\title{
Lucha contra la desinformación desde las bibliotecas universitarias
}

\section{Fighting disinformation from academic libraries}

\author{
Sara Martínez-Cardama; Laura Algora-Cancho
}

Cómo citar este artículo:

Martínez-Cardama, Sara; Algora-Cancho, Laura (2019). “Lucha contra la desinformación desde las bibliotecas universitarias". El profesional de la información, v. 28, n. 4, e280412.

https://doi.org/10.3145/epi.2019.jul.12

Artículo recibido el 09-01-2019

Aceptación definitiva: 20-05-2019

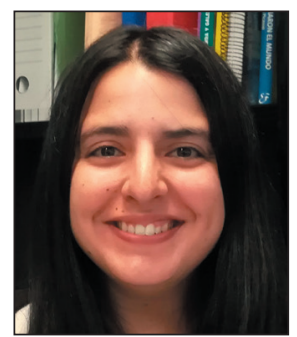

Sara Martínez-Cardama $\square$

https://orcid.org/0000-0001-7035-5884

Universidad Carlos III de Madrid

Departamento de Biblioteconomía y

Documentación

Instituto Agustín Millares

Madrid, 128.

28903 Getafe (Madrid), España

smarti1@bib.uc3m.es

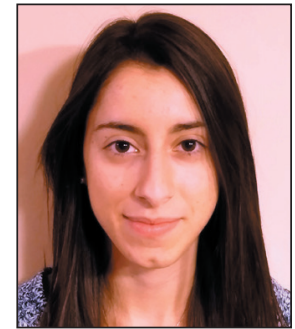

Laura Algora-Cancho

https://orcid.org/0000-0002-4984-5077

Universidad Carlos III de Madrid

Departamento de Biblioteconomía y

Documentación

Madrid, 128.

28903 Getafe (Madrid), España

laura_algora@hotmail.com

\section{Resumen}

Se analiza el papel potencial que tienen las bibliotecas universitarias para concienciar sobre las noticias falsas y su problemática. Se lleva a cabo un estudio que permite conocer la actual labor y acciones desarrolladas por las bibliotecas universitarias españolas respecto a este fenómeno. Se aplicó un cuestionario a las 75 universidades tanto públicas como privadas existentes en España. La tasa de respuesta fue del $56 \%$. Los resultados muestran preocupación por el fenómeno de la desinformación en la educación superior y destacan el necesario papel de las bibliotecas como agentes infomediarios. Este estudio constituye una aproximación basada en datos sobre la actual relación de las bibliotecas universitarias y el fenómeno de la posverdad en el marco bibliotecario español.

\section{Palabras clave}

Posverdad; Noticias falsas; Desinformación; Redes sociales; Medios; Bibliotecas universitarias; Bibliotecas académicas; Alfabetización informacional; Infomediación; España.

\begin{abstract}
The potential role of university libraries in raising awareness about fake news and its problems is addressed. For this, a study that allows to know the current work and actions developed by the Spanish university libraries regarding this phenomenon was carried out. A questionnaire was sent to the 75 public and private universities in Spain. The response rate was $56 \%$. The results show concern about the phenomenon of disinformation in higher education and highlight the necessary role of libraries as infomediarian agents. This study is an approximation based on data on the current relationship between university libraries and the phenomenon of post-truth in the Spanish library framework.
\end{abstract}

\section{Keyworks}

Post-truth; Fake news; Disinformation; Trust; Social media; Higher education; University libraries; Academic libraries; Media literacy; Information literacy; Infomediation; Spain. 


\section{Introducción}

Uno de los fenómenos actuales que genera más debate y supone un reto para los profesionales de la información y documentación lo constituye el neologismo llamado "posverdad", que describe una realidad en la que los hechos objetivos tienen menor influencia en la opinión pública que criterios apoyados en los sentimientos y emociones. El nuevo entorno tecnológico aupado por los medios sociales utilizados para el consumo de información, favorece la aparición de relatos distorsionados ante cualquier acontecimiento. Este consumo está favorecido por una transmisión rápida del mensaje, gracias a la viralidad y multiplicidad de canales, así como la confusión entre emisor-receptor. Esto provoca la falta de comprensión y profundización en la lectura, así como la pérdida de autoridad en el mensaje informativo.

Las noticias falsas no constituyen más que la punta del iceberg, o la consecuencia de una realidad más compleja que ha sacudido los pilares en los que se sustenta el periodismo tradicional. Éste ve constantemente debatida su calidad y capacidad para luchar contra este fenómeno, mientras que una mayor automatización e infomediación entra a formar parte del ecosistema comunicativo, a través de plataformas de verificación llamadas fact checkers. No obstante, las consecuencias de la posverdad no sólo se limitan al periodismo, sino que afectan a nivel político y educativo.

En este escenario, comienza a ser debatido el papel que han de tener los profesionales de la información y bibliotecas como aliados en este proceso. Las bibliotecas han ejercicio tradicionalmente tareas de infomediación, y ahora, esta intervención es más necesaria que nunca constituyendo un elemento facilitador en este proceso: reubicando y redefiniendo las nuevas alfabetizaciones mediáticas, fomentando el pensamiento crítico y convirtiéndose en un elemento clave en su papel de advocacy (defensa pública) en la sociedad en lo que respecta a este debate que comienza a estar en la agenda política de varios organismos y gobiernos (Caridad-Sebastián et al., 2018).

La preocupación sobre la implicación de las bibliotecas ante este fenómeno es reciente, coincidiendo con el debate generado sobre su influencia en procesos políticos como las elecciones de Estados Unidos o el Brexit en Reino Unido. Las instituciones internacionales con competencias en la materia (IFLA o ALA, en el caso norteamericano) han comenzado a llevar a cabo iniciativas y programas formativos que impulsan el papel de las bibliotecas en este tema.

La bibliografía especializada también muestra el reciente interés por el asunto, mediante:

- investigaciones de tipo teórico en las que se fundamenta esta participación (Rochlin, 2017; Batchelor, 2017);

- contribuciones que fomentan la adaptación de las multialfabetizaciones (Banks, 2016; Johnson, 2017);

- debates que afectan a los pilares de la biblioteconomía, como la neutralidad bibliotecaria (Shores, 2018).

En este interesante abanico de publicaciones comienzan a verse propuestas metodológicas concretas de aplicación en las bibliotecas escolares (Lamb, 2017). Las bibliotecas universitarias también son vistas necesarias para el fomento del pensamiento crítico. Así, trabajos como el de Anderson (2017) ponen el foco en la responsabilidad en la gestión de colecciones, mientras otros proponen acciones a nivel de grado (Rose-Wiles, 2018). Existe todavía una ausencia de trabajos de campo que analicen la posición de las bibliotecas universitarias ante este hecho, y menos aún en España. Teniendo en cuenta esta premisa, el objetivo del artículo es identificar las acciones existentes, así como las principales líneas que se pueden adoptar en el futuro por parte de las bibliotecas universitarias.

\section{Revisión bibliográfica}

\subsection{Contexto de las noticias falsas}

Los neologismos "posverdad" y "noticias falsas" han ocupado las páginas de los medios en los últimos años, dibujando un nuevo mapa informativo, político y social. Ambos fueron elegidos palabras del año por los diccionarios Oxford y Collins en 2016 y 2017 (Oxford Dictionaries, 2016; Collins Dictionary, 2017). Precisaremos las diferencias entre ambos.

Tal y como señala Corner (2017), la posverdad supone un cambio social que afecta a la manera de hacer política y a cómo tratan la información los medios. Por su parte, el concepto de noticias falsas se aplica a entornos mediáticos asociados por su rapidez y viralidad. Debido que ambos términos tratan de calificar un fenómeno complejo, la Comisión Europea a través de su grupo de expertos recomendó utilizar el concepto "desinformación" frente al de noticias falsas, considerado más reduccionista.

Para categorizar este fenómeno, Caridad-Sebastián et al. (2018) establecieron tres elementos que pueden resumir las causas del auge del contexto actual de la desinformación:

1) los nuevos hábitos en el acceso y uso de la información: marcado por un aumento de la obtención de información por redes sociales y Whatsapp, principal foco de propagación de noticias falsas. El comportamiento informacional en estos medios se caracteriza por la viralidad e inmediatez. A esto hay que añadir el componente emocional del actual acceso y uso a la información online (Cooke, 2017). Esta autora manifiesta que la sobrecarga informativa existente en los medios sociales fuerza al individuo a una búsqueda de información más simple, generalmente apoyada por fuentes menos fiables. Asimismo, este tipo de informaciones están afectadas por un componente emocional muy marcado, por lo que su capacidad de expansión es mayor; 
2) el contexto tecnológico: marcado por la presentación personalizada de los resultados. Las teorías denominadas "filtros burbuja" (filter bubbles) y "cámaras de resonancia" (echo chambers) facilitan que los usuarios encuentren información que refuerce y retroalimente sus creencias, sin que ésta pueda rebatir convencimiento previo;

3) el contexto social y político está marcado por la polarización existente y el creciente descrédito en los medios tradicionales. En este clima polarizado, la información falsa se difunde más rápidamente y afecta de manera más intensa que las noticias verídicas: solo en la red social Twitter, las noticias falsas han tenido un $70 \%$ más de retweets que las verdaderas (Vosoughi; Roy; Aral, 2018).

La información científica no es ajena a este fenómeno y también sufre sus consecuencias. La pseudociencia (fake science) llena las redes sociales, y verdades científicas como el cambio climático son discutidas y contrarrestadas. En Sanidad esta problemática puede ser muy perjudicial, pues en muchas ocasiones se divulgan informaciones falsas relacionadas con medicamentos, enfermedades o soluciones alternativas, poniendo en riesgo la salud de las personas. En España, se ha creado la iniciativa \#SaludSinBulos, una plataforma para denunciar y combatir las noticias falsas en materia de salud, presentada por la Asociación de Investigadores en eSalud (AIES).

En la categorización de las noticias falsas es preciso alejarse de la discusión de verdadero y falso, ya que, en ocasiones, una noticia falsa no es falsa al $100 \%$ sino que lleva implícita gran subjetividad o una distorsión de los hechos. Ante la complejidad de este hecho, Zimdars (2016), al frente del recurso colaborativo Open Sources, realizó 12 categorizaciones no excluyentes entre sí, que abarcan desde información falsa, sátira hasta las denominadas pseudociencias (fake science) o las noticias que incitan al odio, sin olvidar los titulares clickbait, sensacionalistas y tendenciosos, para la obtención de más ingresos a través del incremento de clics.

La tecnología y la inteligencia artificial están ayudando en la detección de noticias falsas a través de mecanismos y plataformas de verificación basados en el aprendizaje automático y el análisis textual (Álvaro, 2017). Las redes sociales también se han sumado a esta lucha, después de que Facebook fuera duramente criticado por permitir que informaciones falsas se difundiesen en su red social durante las elecciones presidenciales de Estados Unidos, así como por la presencia de algoritmos que favorecen la presencia de burbujas filtro. Posteriormente, tanto Facebook como Google se comprometieron a restringir la publicidad de los sitios web falsos, además de dificultar la divulgación de este tipo de noticias y utilizar software verificador (IFLA, 2017). Facebook ha comenzado a introducir mecanismos para la valoración de las fuentes fiables en su sección de noticias. Mientras tanto, Google ya ha implementado un

Una de las consecuencias de la posverdad informativa, es la necesaria adquisición de competencias digitales de los mediadores profesionales

comprobador de datos para cuando los usuarios buscan información sobre un tema controvertido. Para que estos sean considerados deberán cumplir con el uso de Schema.org ClaimReview en las páginas en donde se analizan declaraciones públicas (Summers, 2017). Sin embargo, en este escenario de automatización, la mediación comunicativa es más necesaria que nunca, ya que los fact checkers no pueden ser meros repositorios de datos sin contexto ni capacidad de generar conocimiento (Caridad-Sebastián et al., 2018). Así pues, una de las consecuencias de la posverdad informativa, es la necesaria adquisición de competencias digitales de los mediadores profesionales.

En este sentido, el fomento de competencias digitales y el buen uso de internet es clave para generar una mayor conciencia. Cabe destacar la buena labor de organizaciones sociales como la británica doteveryone.org.uk en su lucha por un uso de internet más justo y responsable. Esta es la línea que a nivel internacional se considera principal para la lucha contra las noticias falsas. Así, la Comisión Europea creó en enero de 2018 un grupo de expertos de alto nivel para aconsejar sobre las iniciativas políticas para hacer frente a las noticias falsas y la desinformación. Como resultado se publicó el informe A multi-dimensional approach to desinformation con el objetivo de examinar las mejores acciones según los principios fundamentales de la Unión Europea. En el informe se recogen las siguientes recomendaciones (Comisión Europea, 2018):

- mejorar la transparencia de las noticias online;

- promover la alfabetización informacional y mediática, así como ayudar a los usuarios a navegar correctamente a través de los medios digitales;

- elaborar aplicaciones tecnológicas que permitan empoderar tanto a usuarios como periodistas a la hora de enfrentar la desinformación;

- garantizar la diversidad y sostenibilidad del ecosistema de medios europeos;

- promover la investigación sobre el impacto de la desinformación, evaluando las medidas realizadas desde diferentes sectores.

A lo largo del informe se desprende que el fomento de la educación y la investigación prevalece sobre otras medidas más restrictivas como las penalizaciones o el fomento de la legislación que frene la desinformación. Esto constituye un debate complejo, ya que choca con principios básicos como la libertad de expresión por lo que muchos estados han declinado, por el momento, optar por la vía regulatoria. 


\subsection{Bibliotecas universitarias y noticias falsas: acciones desarrolladas}

A raíz de los últimos acontecimientos políticos y los problemas causados por las noticias falsas y la desinformación, se ha debatido acerca de la necesidad cada vez más imperiosa de que las bibliotecas actúen como mediadoras en el proceso.

Las grandes asociaciones bibliotecarias con competencias en la materia están dando un paso al frente para reforzar el papel de las bibliotecas ante este nuevo escenario. Con el fin de que los usuarios tengan una mayor concienciación de este problema, la International Federation of Library Associations and Institutions (IFLA), principal organismo internacional del sector bibliotecario, presentó una infografía sobre cómo detectar noticias falsas, diseñada a partir de los métodos impulsados por FactCheck.org (figura 1). Debido a su éxito y repercusión se ha traducido a 37 idiomas, además de ser expuesta en medios de comunicación. Por su parte, la American Library Association (ALA) contribuye al fomento del pensamiento crítico en torno a las noticias falsas a través de seminarios web y guías temáticas para dotar a los bibliotecarios estadounidenses de herramientas de identificación de fuentes fiables de noticias. Asimismo, en su papel de advocacy, lleva a cabo la campaña Libraries transform para promover la conciencia pública sobre el valor, impacto y servicios proporcionados por las bibliotecas y sus profesionales. Su división para bibliotecas universitarias, Association of College \& Research Libraries (ACRL), trata de integrar las actividades dentro del nuevo marco ACRL Framework for information literacy (2016).

A pesar de que en un principio se puso el foco en el papel destacado de bibliotecas escolares y públicas, las bibliotecas universitarias constituyen un engranaje clave en la ¿ESTA NOIICIA ES FALSA?

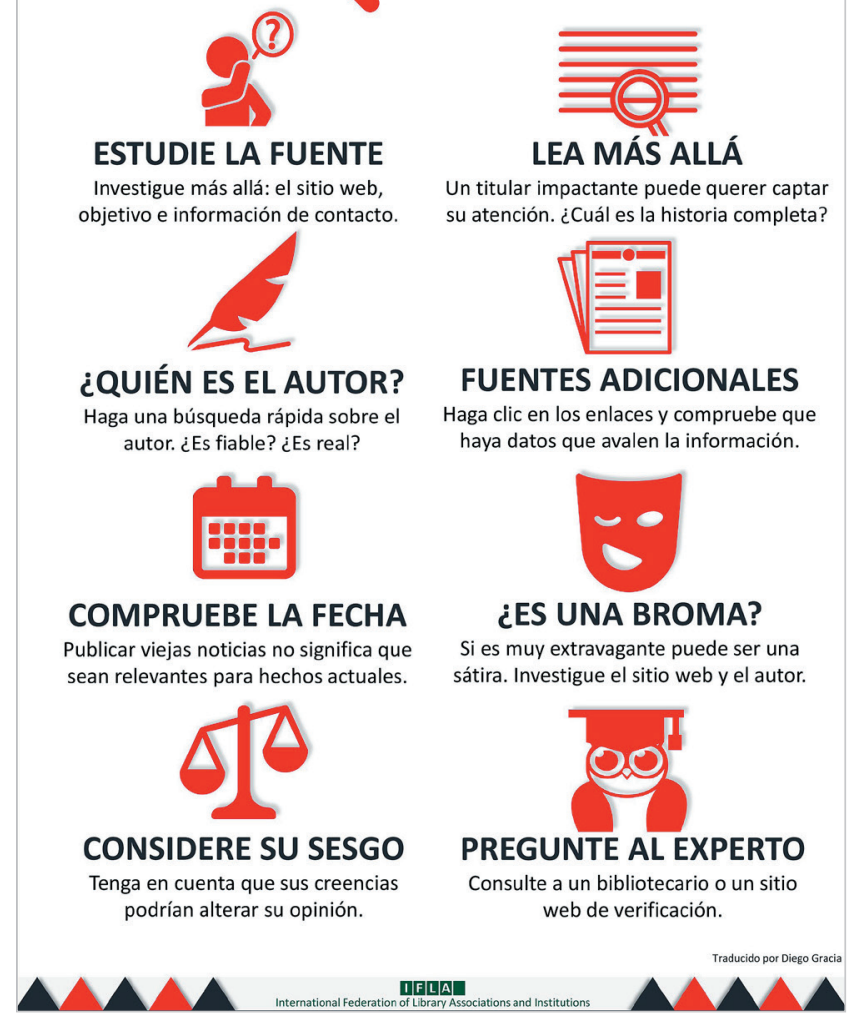

Figura 1. Infografía de la IFLA con pautas para detectar noticias falsas lucha contra la desinformación ya que sus efectos llegan a colectivos como el universitario y afectan a la comunicación científica. Así lo ha constatado el estudio Evaluating information: The cornerstone of civic online reasoning, realizado por el Stanford History Education Group (SHEG, 2016) de la Stanford University, que determinó que la mayoría de los estudiantes tiene dificultades para juzgar la veracidad de la información que se publica en internet, por lo que son fácilmente engañados por los contenidos publicitarios. También se mostró la incapacidad del alumnado para distinguir anuncios de artículos de noticias y determinar de dónde procedía la información.

Los estudiantes, por tanto, representan un colectivo crítico a la hora de verse afectados por esa problemática debido a la elevada utilización de medios sociales y difusión de contenidos virales. La biblioteconomía académica puede dar respuestas efectivas y, desde nuestra perspectiva, más acordes a la realidad informativa de los estudiantes. El grueso de las aportaciones actuales las resumen Finley, McGowan y Kluever (2017) en tres pilares:

- gestión de la colección digital;

- instrucción bibliotecaria (alfabetización informacional);

- papel de defensa pública (advocacy) en la sociedad.

En cuanto a la primera, el riesgo de una interferencia en la gestión de colecciones atenta contra el principio de neutralidad ideológica, aunque este punto está siendo debatido por la llamada biblioteconomía crítica (critical librarianship) que defiende posicionamientos más activos de cara a la defensa del bien común y la democracia. En cuanto a las bibliotecas universitarias, el debate no se genera tanto en la selección de información académica sino en la necesaria colaboración con proveedores de información digital para que visibilicen mejor los recursos fiables y eviten la inclusión de contenido falso o tendencioso. Rose-Wiles (2018) se refiere en el contexto académico expresamente a las revistas depredadoras (predatory journals) algunas de las cuales están indexadas en bases de datos. Esta autora llama a la necesaria cooperación entre bibliotecarios, proveedores y editores para favorecer el acceso a fuentes cuidadosamente "curadas" y seleccionadas.

No obstante, el gran pilar sobre el que pivotan las actividades realizadas por parte de las bibliotecas universitarias es la alfabetización informacional, a través de dos vías:

- la implicación directa del personal bibliotecario en la formación;

- la elaboración de guías de recursos temáticos, que, al margen de seleccionar contenidos, incluyen actividades que interpelan directamente al estudiante evaluando su sesgo de confirmación como en las bibliotecas de Berkeley o de la University of Illinois. 
Se ha detectado que, a pesar de los esfuerzos, la actual formulación de la alfabetización informacional no llega para abarcar este fenómeno, debido al propio comportamiento emocional informativo de los estudiantes, que está presente en su vida cotidiana y se traslada al aula. Así, estrategias basadas en la aplicación de listas de evaluación de recursos como el test Currency, relevance, authority, accuracy, and purpose (Craap) no son suficientes, porque las nuevas competencias infocomunicacionales han de ser más intrínsecas y actuar de manera más rápida, a la misma velocidad que circula la información. Farkas (2018) alude a que difícilmente un individuo va a aplicar en su vida real todos los criterios, debido a la forma en la que se consume la información. Asimismo, los investigadores del Stanford History Education Group señalaban en su estudio que estas checklists no están acordes a una aplicación real de evaluación de fuentes, y Caulfield en su libro Web literacy for student fact checkers (2017) presenta mecanismos de evaluación más rápidos y asociados al fact checking en entornos que los estudiantes conocen y están familiarizados, como contenidos virales en medios sociales.

Sin embargo, la revisión de la bibliografía muestra que sin la colaboración biblioteca-profesorado cualquier iniciativa de alfabetización informacional que se realice, no tendrá la capacidad de perdurar en el tiempo, por lo que se demandan modelos más flexibles de difundir las fuentes a través de la docencia reglada.

\section{Metodología del estudio}

Se llevó a cabo una investigación de tipo cuantitativo basada en una encuesta a la totalidad del sistema universitario español. Para ello se identificaron las bibliotecas de todas las universidad públicas y privadas a través del directorio de la Red de Bibliotecas Universitarias (Rebiun). Tras averiguar los datos de contacto, los 75 responsables de estas bibliotecas fueron invitados a participar en el estudio. De ellos, respondió un total de 42 (56\%).

Se eligió crear un cuestionario ad-hoc en Google Forms, que fue enviado junto con la explicación del proyecto y el objeto de la investigación, y estuvo disponible desde marzo a junio de 2018. Para la elaboración de las preguntas se tuvieron en cuenta los estudios de Finley, McGowan y Kluever (2017), Rochlin (2017) y Batchelor (2017), entre otros.

Se creó un cuestionario mixto y autoadministrado, que incluyó 10 preguntas abiertas y cerradas. En lo que respecta a estas últimas se eligieron los formatos de respuesta dicotómica y múltiple. Las dimensiones y estructura interna del cuestionario la componen tres bloques:

- el primer grupo de preguntas trata de conocer la percepción como bibliotecarios del papel que las bibliotecas han de tener en las noticias falsas y los mecanismos que existen para combatirlas;

- el segundo se centró en los riesgos de la verificación de la información académica por parte de los estudiantes en los centros;

- la última parte recopiló las iniciativas actuales o futuras que se llevan a cabo.

Para facilitar la interpretación de los resultados, en la tabla 1 se recogen las dimensiones del cuestionario y sus variables de estudio.

Tabla 1. Variables de estudio del cuestionario

\begin{tabular}{|c|c|}
\hline Sección del cuestionario & Ámbitos de evaluación \\
\hline $\begin{array}{l}\text { 1. Percepción sobre las noticias falsas y la validez } \\
\text { de los mecanismos que existen para combatirlas }\end{array}$ & $\begin{array}{l}\text { 1.1. Papel relevante de las bibliotecas ante las noticias falsas. } \\
\text { 1.2. Técnicas o actividades a través de las que podría solucionarse el fenómeno de la desin- } \\
\text { formación que causan los bulos y noticias falsas en internet. }\end{array}$ \\
\hline $\begin{array}{l}\text { 2. Riesgos sobre la verificación de la información } \\
\text { académica por parte de los estudiantes }\end{array}$ & $\begin{array}{l}\text { 2.1. La desinformación afecta tanto a las noticias en los medios de comunicación (a nivel } \\
\text { político, social, económico...) como a la información científica. } \\
\text { 2.2. Las redes sociales potencian la distribución de información científica no verificada. } \\
\text { 2.3. Los estudiantes universitarios pueden verse afectados a nivel académico por la dificul- } \\
\text { tad de identificar la veracidad de la información. } \\
\text { 2.4. Papel de la alfabetización mediática e informacional como método para combatir las } \\
\text { noticias falsas y su problemática. } \\
\text { 2.5. Tipos de recursos a través de los cuales la biblioteca puede apoyar este nuevo tipo de } \\
\text { alfabetizaciones mediáticas. }\end{array}$ \\
\hline 3. Iniciativas presentes o futuras & 3.1 Iniciativas presentes o futuras. \\
\hline 4. Pregunta abierta & 4.1 Información de interés o relevante no contemplada en los items anteriores. \\
\hline
\end{tabular}

\section{Resultados y discusión}

La tasa de respuesta ha sido muy variada respecto a la ubicación y al tipo. Se cuenta con bibliotecas universitarias de prácticamente todas las comunidades autónomas (a excepción del Principado de Asturias, La Rioja y Aragón) y con respuestas de 29 bibliotecas de universidades públicas y 13 de universidades privadas (figura 2). 


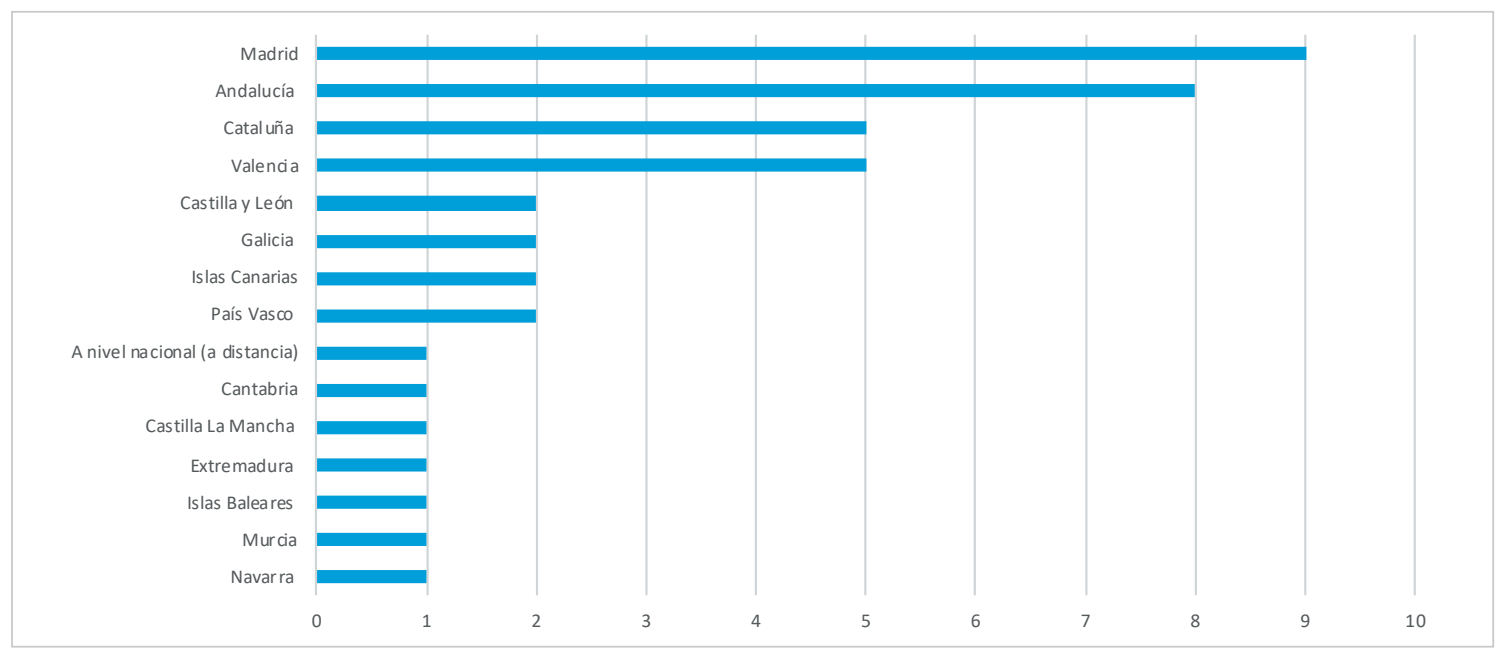

Figura 2. Distribución territorial de las respuestas en función del número de universidades

Los principales resultados del estudio se muestran estructurados en las tres áreas de interés que conforman el cuestionario.

\subsection{Percepción sobre las noticias falsas y la validez de los mecanis- mos que existen para combatirlas}

Ante la pregunta de si las bibliotecas han de tener un papel clave en la lucha contra las noticias falsas, un $88 \%$ de las respuestas de las bibliotecas universitarias españolas es afirmativa. De las restantes, el $5 \%$ considera que no es un agente válido para combatirlas, mientras que el $7 \%$ no sabe o no contesta.

Con respecto a las técnicas mediante las que podrían enfrentarse a la difusión de estas noticias y su consecuente dimensión social, los responsables de las bibliotecas consideraron, ante una pregunta de respuesta múltiple, las respuestas que se muestran en la figura 3.

Se dota de mayor importancia a las respuestas asociadas con la formación y la educación (38\% y $36 \%$ respectivamente). Para ello, consideraron clave la colaboración de la biblioteca universitaria y el claustro de profesores en la realización de actividades conjuntas que puedan ser aplicadas. La tecnología adquiere el siguiente nivel de importancia con un $29 \%$. Las respuestas que priman la opción de las leyes o regulaciones, son minoritarias (10\%). Las respuestas dadas por los responsables de las bibliotecas universitarias van en la línea de las tendencias encontradas en la bibliografía y de los informes internacionales como el de la Comisión Europea al considerar las leyes o regulaciones como una opción menos indicada. El 52\% de los encuestados considera este problema como una realidad compleja que no puede ser abordada con una única vía de acción.

\subsection{Riesgos sobre la verificación de la información académica por parte de los estudiantes}

Ante la pregunta que planteaba si consideraban que la desinformación y noticias falsas son un hecho que afecta sólo a las noticias publicadas en medios de comunicación (ya sea a nivel económico, político o social) o por el contrario también la información científica se ve de igual modo alterada, el 79\% de los responsables opinaron que ambas están sujetas al mismo riesgo. Esto es un hecho relevante, muy en la línea de los acontecimientos actuales en los que la poca cultura científica hace que se difundan informaciones de estudios sesgados y con falta de rigor. Así, por ejemplo, bibliotecas de universidades de Estados Unidos (Yale, UCLA...) y Reino Unido (Manchester) comienzan a incluir en sus guías temáticas sobre acceso abierto listas de editoriales sospechosas, poco recomendables, o directamente, depredadoras. Estas guías temáticas difunden infografías y checklists para detectar este tipo de revistas o editoriales. Entre ellas destaca la web: https://thinkchecksubmit.org 
Las bibliotecas deben tomar conciencia de que la información falsa o tendenciosa no sólo se transmite en medios sociales, sino que los tradicionales canales académicos pueden contener ciertas prácticas ilícitas, y pueden prevenir a la comunidad sobre ellas.

En cuanto a las preguntas que ponen el foco en los estudiantes, nicho crítico que puede sufrir los efectos de la desinformación, el $91 \%$ de los bibliotecarios considera que las plataformas digitales potencian la difusión de estas noticias, especialmente las redes sociales. Por ello son el medio hacia el que deben reformularse las competencias infocomunicacionales para que la capacidad de evaluación de fuentes sea más dinámica y adaptada a estos canales de información. Esta capacidad de verificación, o fact checking, debe desarrollarse primero en este entorno, ya que el $88 \%$ de las bibliotecas considera que los estudiantes sí pueden verse perjudicados académicamente por la dificultad que les supone la identificación de la veracidad de la información. Las bibliotecas universitarias y los docentes deben ser conscientes de que la información para los estudiantes no sólo existe en el entorno académico, por lo que los vicios o malas prácticas adquiridas en medios sociales pueden verse trasladadas a prácticas curriculares.

Los recursos o actividades que la biblioteca debería desarrollar fueron contestados a través de una respuesta múltiple (figura 4).

Las respuestas procedentes de las bibliotecas universitarias están alineadas con las tendencias detectadas en la bibliografía y consideran las guías temáticas la opción prioritaria para promover estas competencias (91\%). Estas guías monográficas sirven tanto para explicar los problemas asociados a las noticias faltas (filtros burbujas, cámaras de resonancia), como para la difusión de criterios de evaluación, herramientas de verificación y una selección de recursos para su consulta. Estos recursos son tanto documentación de apoyo como una oportunidad para difundir nuestra propia colección.

En segundo lugar destaca el uso de material gráfico como infografías. Éstas son un recurso muy potente y utilizado en varias guías temáticas para explicar distintos procesos asociados con la desinformación, o los pasos esenciales para la verificación de fuentes. Asimismo, de manera expresa, la difusión de la infografía de la IFLA es útil para llamar la atención sobre esta problemática.

Por último, con la misma frecuencia (74\%) destacan las opciones de formación como cursos o tutoriales interactivos donde se demuestren sus sesgos y se oriente. Al mismo nivel está la realización de campañas en redes sociales, de manera activa. En este sentido, la biblioteca puede difundir la problemática incluyendo en sus contenidos infografías o publicaciones relacionadas de otras instituciones u organizaciones. Sin embargo, habría que plantearse si la biblioteca universitaria podría ir más allá y actuar de verificador (fact checker) de información científica en medios sociales. En concreto una manera de que pueda aumentar su visibilidad sería conocer qué bulos o estudios son debatidos en la Red sobre disciplinas del campus y sumarse a desmentirlos difundiendo a la vez su colección física o virtual.

\subsection{Iniciativas actuales o futuras en las bibliotecas universitarias españolas}

A la pregunta de si las bibliotecas universitarias españolas realizan o plantean realizar alguna de las actividades anteriormente mencionadas, sólo un $52 \%$ de los directores de las bibliotecas contesta afirmativamente, lo que supone poco más de la mitad. Sin embargo, se considera algo positivo, teniendo en cuenta la ausencia de directrices o recomendaciones específicas por parte de organismos con competencias en la materia como Rebiun.

Con respecto a esta pregunta, se formuló otra de respuesta abierta para saber cuáles son las acciones que se están tomando desde las bibliotecas universitarias que afirmaron que tienen actuaciones previstas en materia de desinformación y noticias falsas. Estas respuestas se sistematizaron mediante un sistema de categorías creadas ad hoc (tabla 2). 
Tabla 2. Categorización de las actividades sobre noticias falsas y desinformación por parte de bibliotecas universitarias españolas

\begin{tabular}{|c|c|}
\hline Categorías & Actividades \\
\hline 1. Actividades formativas & $\begin{array}{l}\text { 1.1. Actividades específicas. } \\
\text { Cursos de formación en alfabetización informacional. } \\
\text { Formación relacionada con la evaluación de la información. } \\
\text { Cursos sobre competencias digitales dirigidos a alumnos de grado y master. } \\
\text { Inclusión de un módulo específico en las sesiones de formación a alumnos recién llegados a la } \\
\text { Universidad. }\end{array}$ \\
\hline 2. Actividades de difusión & $\begin{array}{l}\text { 2.1. Difusión de la infografía de la IFLA sobre cómo detectar noticias falsas o de información } \\
\text { gráfica en las instalaciones. } \\
\text { 2.2. Actividades de difusión en redes sociales: } \\
\text { Campañas a través de las redes sociales sobre noticias falsas y desinformación. } \\
\text { Uso de la plataforma digital Pinterest para la publicación de material visual sobre posverdad, } \\
\text { desinformación y noticias falsas. }\end{array}$ \\
\hline 3. Actividades audiovisuales & 3.1. Publicación de vídeos en el canal de YouTube de la biblioteca. \\
\hline 4. Actividades de información o referencia & $\begin{array}{l}\text { 4.1. Guías temáticas. } \\
\text { 4.2. Colecciones monográficas relacionadas con el tema estudiado. }\end{array}$ \\
\hline
\end{tabular}

En este sentido, cabe señalar que, salvo algunas actividades específicas orientadas a las noticias falsas, muchas de ellas tienen una orientación más general y se encuadran en acciones generales de alfabetización informacional.

Por último, se dejó una pregunta abierta que reflejase sus posibles dudas, sugerencias o comentarios. En ella varios profesionales recalcan la necesidad de una mayor colaboración profesorado-biblioteca para poder desarrollar estas actividades. Señalan que sólo de esta manera se podrán llevar a cabo propuestas efectivas al respecto. Por otro lado recomiendan la difusión de informes que guíen la instrucción bibliotecaria ante este fenómeno, en concreto Combating fake news in the digital age de Burkhardt (2017). Esto es un indicio de que las bibliotecas siguen la problemática de este fenómeno, la cual puede parecer ajena o estar más vinculada a un ámbito político o social.

\section{Conclusiones}

El presente estudio recoge aportaciones tanto para las bibliotecas universitarias españolas como para aumentar el conocimiento e investigación dentro de la biblioteconomía académica en lo que a la problemática sobre noticias falsas se refiere, un terreno donde todavía son pocas las iniciativas.

En primer lugar, muestran una relación positiva entre las tendencias detectadas en la revisión de la bibliografía y algunas de las iniciativas que las bibliotecas universitarias españolas comienzan a desarrollar.

En referencia a las líneas de acción, se ha valorado de manera esencial la formación en nuevas competencias digitales. Así, casi la totalidad de los entrevistados enmarca las actividades a desarrollar dentro de la alfabetización informacional. Las autoras consideran que ésta debe adaptarse a la nueva realidad: no sólo debe proveer herramientas para evaluar fuentes o desarrollar hábitos de evaluación en la Web sino orientarse a un terreno, el de los medios sociales, en el que los usuarios de información han de tomar decisiones rápidas sobre en qué confiar. Se trata de adquirir habilidades intrínsecas para moverse en un área compleja como el de los actuales proveedores de información. La alfabetización informacional debe abordar ese escenario y ser capaz de llevarlo al terreno académico. Para ello, la colaboración biblioteca-profesorado es esencial para que pueda trasladarse a diferentes asignaturas o etapas de la vida académica de los estudiantes: utilizar discursos en Twitter sobre un tema de actualidad o controvertido ayuda a analizar las voces fiables, así como los sesgos que pueden producirse. Por ello, aprender a verificar un perfil original, a detectar un bot, rastrear la veracidad de una noticia en internet, realizar búsquedas inversas para verificar imágenes o vídeos deben ser actitudes inherentes para cualquier estudiante.

La elaboración de guías temáticas se considera el recurso más valorado por parte de las bibliotecas. Esa acción es destacada, pues puede facilitar un conocimiento de cómo puede afectar la desinformación a su vida cotidiana (trabajo, estudios, redes sociales...) y para los profesionales es una buena oportunidad para difundir recursos de calidad de la biblioteca y de ofrecer un servicio de referencia digital que amplíe los tradicionales recursos sobre evaluación de fuentes. Sin embargo, en nuestra opinión,

\section{Debe haber una colaboración bibliote- ca-profesorado más flexible para defi- nir de manera conjunta actividades que puedan ayudar a estudiantes}

si estas guías se comparten a través de canales educativos como Moodle u otros LMS, y se emplean como recurso para la docencia, aumentaría su impacto y permitiría ver a la biblioteca como un punto de información y asesoramiento especializado en los mecanismos que la sociedad dispone para frenar la plaga de las noticias falsas.

Basándose en las respuestas obtenidas se puede concluir que debe haber una colaboración biblioteca-profesorado más flexible para definir de manera conjunta actividades que puedan ayudar a estudiantes. Asimismo, los docentes e investigadores también están expuestos a los peligros de la desinformación, víctimas de las revistas depredadoras (predatory journals) y métricas engañosas (misleading metrics). 


\section{Notas}

1. Encuesta disponible a través del siguiente URL: https://bit.ly/2VusPFs

\section{Referencias}

ACRL (2016). Framework for information literacy for higher education. Association of College and Research Libraries. http://www.ala.org/acrl/standards/ilframework

Álvaro, Sandra (2017). "Noticias falsas: compartir es curar". CCCB LAB. Investigación e innovación en cultura, 7 marzo. http://lab.cccb.org/es/noticias-falsas-compartir-es-curar

Anderson, Rick (2017). "Fake news and alternative facts: five challenges for academic libraries". Insights, v. 30, n. 2, pp. 4-9. https://doi.org/10.1629/uksg.356

Banks, Marcus (2016). "Fighting fake news: How libraries can lead the way on media literacy". American libraries, 27 Dec. https://americanlibrariesmagazine.org/2016/12/27/fighting-fake-news

Batchelor, Oliver (2017). "Getting out the truth: The role of libraries in the fight against fake news". Reference services review, v. 45, n. 2, pp. 143-148.

https://doi.org/10.1108/RSR-03-2017-0006

Burkhardt, Joanna M. (2017). "How fake news spread: Combating fake news in the digital age". Library technology reports, v. 53, n. 8, pp. 10-13.

https://journals.ala.org/index.php/ltr/article/view/6498

Caridad-Sebastián, Mercedes; Morales-García, Ana-María; Martínez-Cardama, Sara; García-López, Fátima (2018). "Infomediación y posverdad: el papel de las bibliotecas". El profesional de la información, v. 27, n. 3, pp. 891-898. https://doi.org/10.3145/epi.2018.jul.17

Caulfield, Mike (2017). Web literacy for student fact-checkers. https://webliteracy.pressbooks.com

Collins Dictionary (2017). "The Collins word of the year 2017 is... Fake news". Collins dictionary. https://www.collinsdictionary.com/word-lovers-blog/new/collins-2017-word-of-the-year-shortlist,396, HCB.html

Comisión Europea (2018). Final report of the High Level Expert Group on Fake News and Online Disinformation. https://ec.europa.eu/digital-single-market/en/news/final-report-high-level-expert-group-fake-news-and-online-disinformation

Cooke, Nicole A (2017). "Posttruth, truthiness, and alternative facts: Information behavior and critical information consumption for a new age". The library quarterly, v. 87, n. 3, pp. 211-221.

https://doi.org/10.1086/692298

Corner, John (2017). “Fake news, post-truth and media - political change". Media, culture \& society, v. 39, n. 7, pp. $1100-1107$. https://doi.org/10.1177/0163443717726743

Farkas, Meredith (2018). "Beyond fake news: Determining what sources to trust". American libraries, 1 June. https://americanlibrariesmagazine.org/2018/06/01/beyond-fake-news

Finley, Wayne; McGowan, Beth; Kluever, Joanna (2017). "Fake news: An opportunity for real librarianship". ILA reporter, v. 35, n. 3, pp. 8-12.

https://goo.gl/i4C1BV

IFLA (2017). "Alternative facts and fake news. Verifiabiblity in the information society". IFLA.

https://blogs.ifla.org/lpa/2017/01/27/alternative-facts-and-fake-news-verifiability-in-the-information-society

Johnson, Ben (2017). "Information literacy is dead: The role of libraries in a post-truth world". Computers in libraries, v. 37 , n. 2. http://www.infotoday.com/cilmag/mar17/Johnson--Information-Literacy-Is-Dead--The-Role-of-Libraries-in-a-Post-TruthWorld.shtml

Lamb, Annette (2017). "Fact or fake? Curriculum challenges for school librarians”. Teacher librarian, v. 45, n. 1, pp. $56-63$. https://goo.gl/QeMdSD

Oxford Dictionaries (2016). "Word of the year 2016 is... 'post-truth'”. English Oxford Living Dictionaries. https://languages.oup.com/press/news/2016/12/11/WOTY-16

Rochlin, Nick (2017). "Fake news: belief in post-truth". Library hi tech, v. 35, n. 3, pp. 386-392.

https://doi.org/10.1108/LHT-03-2017-0062 
Rose-Wiles, Lisa (2018). "Reflections on fake news, librarians, and undergraduate research". Reference \& user services quarterly, v. 57, n. 3, pp. 200-204.

https://doi.org/10.5860/rusq.57.3.6606

Shores, Mark (2018). "The alert collector: Collection development in an era of "fake news". Reference \& user services quarterly, v. 57, n. 3, pp. 176-177.

https://doi.org/10.5860/rusq.57.3.6601

Summers, Nick (2017). “Google will flag fake news stories in search results". Engadget, 4 July. https://www.engadget.com/2017/04/07/google-fake-news-fact-check-search-results/?guccounter=1

Stanford History Education Group (2016). Evaluating information: The cornerstone of civic online reasoning. Palo Alto: Stanford University. https://stacks.stanford.edu/file/druid:fv751yt5934/SHEG\%20Evaluating\%20Information\%20Online.pdf

Vosoughi, Soroush; Roy, Deb; Aral, Sinan (2018). "The spread of true and false news online". Science, v. 359, n. 6380, pp. 1146-1151.

https://doi.org/10.1126/science.aap9559

Zimdars, Melissa (2016). False, misleading, clickbait-y, and/or satirical "news" sources.

https://docs.google.com/document/d/10eA5-mCZLSS4MQY5QGb5ewC3VAL6pLkT53V_81ZyitM/preview

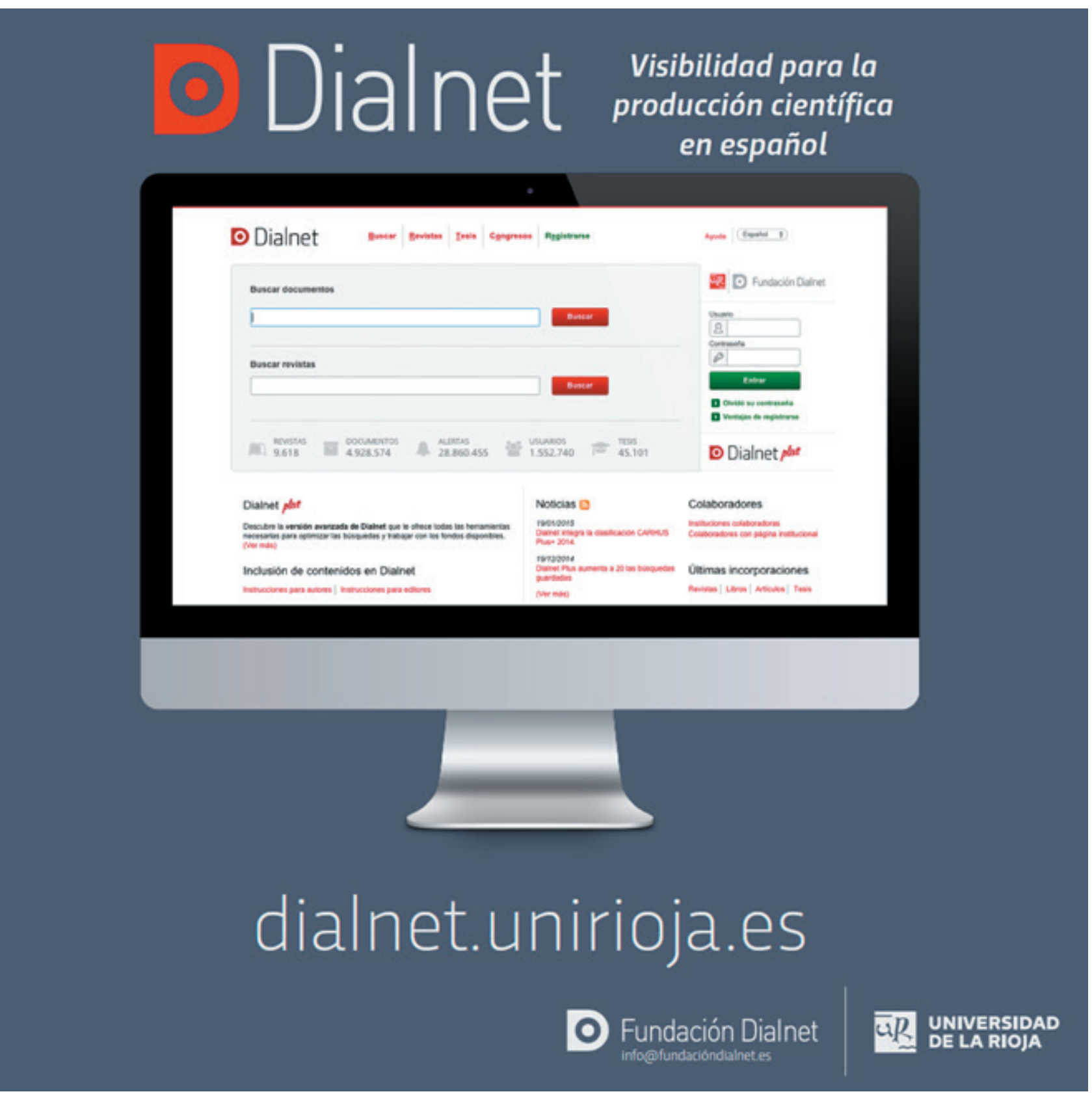

